\title{
Simulating algal bloom in a lake: An interactive multimedia implementation
}

\author{
Robert M. Corderoy, Barry M. Harper and John G. Hedberg \\ Faculty of Education \\ University of Wollongong
}

\begin{abstract}
Many of the software packages presently marketed as simulations are in fact little more than 'pre-set', limited models of the 'real world' systems they are designed to emulate. There is little scope for the user to interact with the model as they would in a 'real world' experience, and this must compromise the intended educational outcomes. The exact nature of what constitutes a 'good simulation' is not agreed upon among researchers or designers alike, but if the goal of the simulation is to provide experiences which approach those in the 'real world', and in so doing, provide opportunity for the development of higher order skills which research in cognitive science is suggesting are important, one must strive for the greatest degree of user interaction as possible.

The achievement of this goal rests with the application of HyperMedia based platforms which may be exploited for their ability to provide the 'genuine interaction' essential to 'real world' systems, across the computeruser boundary. A number of design issues must be addressed if the full potential of HyperMedia based platforms is to be harnessed.
\end{abstract}

Increasingly, educationalists are becoming aware of the worth of computer based simulation as a tool to provide not only support, but in many cases substitute experiences for aspects of curricula which would otherwise be unavailable or difficult to satisfy. Recognition of a niche for such applications of technology in education is not new. It is only now being recognised however, that effective use of the sophisticated technology presently available can only come about with the recognition of a need to develop software in terms of the outcomes of cognitive science research, rather than solely in terms of the capabilities of the hardware itself. 
This paper presents a number of the issues to be considered in the design and development of interactive computer simulations in which the computer acts as an "exploratory tool" (Bliss \& Ogborn 1989), supporting a 'real world' activity. It provides a description of the development of a simulation designed to model algal bloom within a lake which is subject to the effects of input from natural events and the urbanisation of its surrounds. This type of application has the potential to facilitate user understanding of the processes involved in complex and dynamic systems which may otherwise be inaccessible.

The current level of sophistication of interactive multimedia applications provides a vehicle for designers to produce software which fully utilises the capabilities of the available technologies. This is particularly evident in many of the more recent simulation packages, which exhibit a tendency to move away from the earlier simulation format, 'the pre-set model' which provided a very simple approximation of the 'real world' it was trying to mimic. The exact nature of what constitutes 'a true simulation' is not agreed upon amongst researchers or designers alike, but if the goal of the simulation is to provide experiences which approach the 'real world' one must strive to achieve certain design criteria which have their origins in the recognition of the physical capabilities of hypermedia-based applications and in the enhanced understanding of the learning process resulting from current research in cognitive science.

\section{Simulations: Real world substitutes or 'pre-set' experiences}

Simulations have been defined in a number of ways. Martin (1988) has proposed that a simulation is "the dynamic execution or manipulation of a model of an object system for some purpose." Crookall et al. (1987) propose that a simulation "remains defined as a special kind of model representing a 'real world' system, governed by a set of rules".

During the use of such models, the user often comes to see the simulation itself as a 'real world' in its own right. Such models represent systems as either an "in place of" or a "bring to life" format. The question posed by some as to whether the terms 'model' and 'simulation' have similar meanings in this context may prove a pivotal point around which a better understanding of the cognitive outcomes for the users may be achieved. The 'in place of' interpretation or representation applies to the 'standard' notion of a model while, as suggested by (Crookall et al. 1988), both the "in place of" and "bring to life" representations may be applied to simulations. It is the contention of the authors that many of the earlier simulation 
packages were little more than "in place of" mathematical models, presenting the user with limited options in terms of control and outcomes. The interactivity inherent in many of the hypermedia-based simulations currently being produced, provide simulation models which not only enable the user to experience some otherwise inaccessible system, but to "bring it to life" in the sense that the user may interact with, obtain immediate feedback from, and perhaps even alter the underlying model. Such possibilities provide a means of achieving a deeper understanding of the complex interactions and relationships involved in a 'real world' system which may otherwise be beyond their resources.

In categorising simulations, Bell \& Scott (1985) have proposed three types:

- those which are structured

- those which are semi-structured

- and those which are open in form.

It is the latter category which provides the most successful platform for creating 'real life' re-creations of situations and as such provide excellent support for inquiry learning. Although such categorisation is useful, it is a rather simplistic way of making distinctions. Martin (1988) suggests the although there exists an almost infinite array of variations in simulation structure, two trends have evolved in the way in which simulations are perceived. There are those perceived as "in-screen" simulations, characterised by the use of a computer as a modelling device which presents an image of a functioning model bounded by the computer interface. Such simulations are extensively used in educational environments and may be better termed modelling. "Out-screen" simulations are categorised as those which use the computer as a tool to support some essentially 'real world' activity. "This read world activity is the simulation seen as a microcosm of a wider slice of reality"(Martin 1988). Such simulations are characterised by user participation in the sense that the user exercises some degree of control over its operation and possible outcomes. One may also assert that the achievement of a recreation of some 'real world' system is dependent upon convincing the user that the 'boundary of activity' is not the 'black box' (the computer).

\section{General design implications}

A major strength of all computer-based instructional technologies, including simulations and games, rests in their ability to provide motivation, enrichment and elaboration. They allow conceptualisation of cognitive processes and representation in concrete form. "Elaboration cements cognition" (Reigeluth \& Curtis 1987). 
In designing educational materials such as simulations, regardless of the intended audience or level of sophistication one wishes to build in, a paramount concern must always be to provide software which is educationally sound: that is, software which is structured so as to take into account the accepted principles of education and learning.

One can be concerned with two extremes in terms of the parameters which constitute 'good design'. At one end of the continuum, one is concerned with the operation and implications of the technology itself, taking into account such factors as the memory capacity needed, navigational issues, screen design and so on. At the other end of the continuum the concern is that, no matter how good the technology platform, the outcome cannot be educationally effective unless one takes design decisions with reference to the ideas and findings being presented from research in the field of cognitive science with respect to learning theories. Many of the current papers concerned with what constitutes 'good design' fail to examine effectively both issues. Examination of research literature representing both ends of this continuum provides one with unequivocal support for the notion that 'good design' must embrace a carefully considered mix of both approaches, a balanced view.

The process of knowledge acquisition can be improved by designing simulations that support hypothesis formation and problem solving techniques and thus force the user to actively explore the domain. This can only be supported in an environment that has a "feedback" component. The issue of quick "feedback" is a critical aspect of strategies to help in the development of cognitive processes in students. As pointed out by Ohlsson (1987), computers have the potential to carry out moment by moment monitoring of the user. This allows for the continuous adaptation of content and representation, an important aspect in the learning process (Salomon 1981), to meet the changing cognitive needs of the individuals. Loveluck (1989) has proposed that designers should structure simulations and games systems so that the user may switch to a graphic display of the current 'state of play' at any time during the simulation. The essential contribution of this feedback to the effectiveness of a simulation would seem to lie in the aid it provides in visualising the information presented and thus in facilitating further decisions.

The need to develop expertise in students working in computer driven educational environments in particular stems partly from the fact that in most cases, such environments have been developed by experts and as such, are inherently structured around the modus operandi of the expert. The development of expertise requires the acquisition of better organised knowledge. In instructional environments such as simulations, there is therefore a need to provide structuring that ensures students are led more quickly to the kind of organisation that "experts" exhibit. Experts tend to 
store smaller amounts of more general knowledge which requires more reasoning in order to apply it and so, the 'expert mode' involves a higher cognitive load.

In considering the development of expertise or competence, it has been suggested by Decorte (1990) that a number of aspects need to be addressed. Learning is a constructive process and it is the interaction between the learner and the educational environment that allows them to "actively construct" their knowledge. A computer-based environment should have a balance between "discovery learning" (problem solving), "personal exploration" (inquiry learning), and "systematic instruction and guidance" and a well designed simulation should conform to these goals.

An essential function of effective simulations is to provide an environment in which what is learned is controlled by variables which are to a degree, outside the user's control. Among the variables that seem to effect the outcomes of simulations are, navigational issues, and presentation issues (including screen design, sequencing and user monitoring methods). Perception is the foundation of learning and every individual operates at different levels of symbolic perception or processing. One must also consider procedural variables and the internal structure of the system (guided versus unguided instruction and its effect on the development of higher order cognitive skills such as critical thinking and the development of problem solving strategies), which may prove to be a major source of variation in user experiences.

The degree to which a simulation relies on chance to produce output is also a very important aspect. Simulations in which the user is able to input unexpected data and consequently generate unexpected output represent the most useful form of simulation in that such control mimics the 'real world' system. It has been proposed (Gredler 1990) however, that simulations in which the outcomes are largely determined by chance require special consideration during development to avoid certain design errors, including inadvertent reinforcement of inappropriate behaviour, lack of reinforcement for appropriate behaviour and the operation of the concept of "negative utility", whereby the individual is reinforced for a particular action, which in the long term has "punishing consequences" and ultimately leads to "defeat for some users". One must also be cognisant of the need for the content to be 'real world' based if the "participants are to learn the 'right things' " (Bredemeier \& Greenblat, 1981).

Some interesting observations are presented by Laveault \& Corbeil (1990) concerning the learning process occurring in simulations and some of the important aspects of user control and its bearing on the learning process. They outline two models for learning acquisitions in simulations. The first is that proposed by Kryukov \& Kryukova (1986) which suggests that the 
process comprises a series of phases of interaction or dialogue (both direct and intellectual) between the "player and the game". On the other hand, Thatcher's model (1986), suggests that the process involves a series of cycles leading to the acquisition of complexities (rules) of the game. According to Thatcher's model, "all games and simulations are a form of experimental learning". The total experience of a simulation is a series of micro-experiences and repetitions at ever increasingly higher levels. Both of these models assume that there are sequences to the acquisition of learning of the game or simulation and learning from the game or simulation.

In structuring simulations it is important to assess whether the user knows the 'rules' well enough to allow learning to take place. The question to be addressed is, when does the user stop learning about the system itself and start learning from it? Research to date, both cognitively and technically based, would seem to suggest that the sooner this occurs, the better. Ideally a simulation should be structured so as to progress from highly parametered scenarios to less paramatered ones (Thatcher's model of complexity). Kozma (1991) has outlined the importance of the need to place the "minimum load" on "rule learning" so as to free as much "cognitive capacity" as possible to facilitate efficient learning.

The overriding purpose for simulation and modelling systems remains: to provide a substitute experience and the ultimate aim of the developer must be to meet such essential design criteria as:

- Producing a simulation that emulates as closely as possible the 'real world' experience

- Design decisions are based on some appropriate educational paradigm.

- Design decisions are made which are based on a sound knowledge of cognitive science theory.

\section{Hypermedia design issues}

Each of the traditional information processing media (print, audio, and video) have in the past been utilised in educational environments in relative isolation. The multimedia presentation platform appeared and flourished when smaller and more powerful computers became widely dispersed throughout the educational community. This platform permitted each of these media to overlap, thus providing a richness in educational experience never before contemplated. The most recent step in the 'evolution' of computer based educational platforms has been the development of systems in which there exists the capability for 'genuine interaction' and, for the direct and non-linear transfer of information via computer control between multimedia platforms. Such systems are called hypermedia environments. 
Use of the characteristics which empower hypermedia environments to provide the genuine interaction for which they are exploited can produce systems which may have negative outcomes by virtue of their excessive complexity. Consequently, an important concern to be addressed is to ensure that the learning process is supported and not hindered. Hypermedia based systems enable the emulation of the 'expert mode' of learning and, since the general user will not necessarily be an 'expert', the role of cognitive processes must be addressed in order to prevent the complexity of the system 'overcoming' or 'over taxing' the user's processing ability so that cognitive capacity which is otherwise used to 'learn' is 'wasted'. A recurring theme throughout the research literature is the role the computer plays as a tool for lowering the overall work load or, as some researchers including Kozma (1991) and Silver \& Marshall (1990) have suggested, the "cognitive load" involved in processing information. The more this can be achieved, the more the student is then free to concentrate on the acquisition of higher order thinking skills including, planning, evaluation and problem solving or... "executive skills" (Reif, 1987, Schoenfeld 1987).

General issues which need to be considered if one is to ensure the effectiveness and efficiency of hypermedia based platforms in the educational environment include:

- Navigational strategies

- Motivational issues concerning both the information and the technology

- User control

- Perceived relevance

- Self confidence

- User satisfaction.

The objective for both the design and the use of any hypermedia based platforms in educational environments should be centred on identifying these unique attributes and exploiting them to the full in conjunction with the current theories of learning. Whether such unique attributes exist or contribute to the educative process in any special ways is a point of conjecture throughout the literature, however the consensus of opinion would seem to be that the essential aspect to be addressed in the design process is the fitting of such platforms to the theories of cognition and learning, rather than designing and developing platforms for the technologies sake. Decorte (1990) has proposed that "Educators have for too long focused too exclusively on the technology for its own sake".

Navigation "through information space" (Hooper 1988) is now considered as a major issue in the design and function of computer based educational environments. This is particularly true for those based in hypermedia. 
Without a functional navigation system, the user cannot fully exploit the support such platforms provide for exploration based learning environments. The essential feature of an effective functional navigation system is to be simple and intuitive, thus minimising the cognitive load placed on the user. There are numerous suggestions as to the ways in which navigational systems may be improved. Perhaps the most important to be considered include, the use of multiple entry points, the use of guides, the use of partial disclosure and the utilisation of feedback mechanisms that provide information about previous pathways. This latter aspect is of particular interest in terms of the development of critical thinking and other higher order cognitive skills.

\section{A model for Algal Bloom simulation}

\section{Development basic assumptions and design considerations}

The development of a simulation of algal bloom in a lake discussed in this paper represents an enhancement of the educational software package Investigating Lake Iluka, which deals with the general ecology of a lake and its surrounds.

The limiting factors which play a role in the development of an algal bloom in a lake are numerous. Some of these include water quality (turbidity, particulates and the like), temperature, salinity, light levels nutrients, substrate conditions, and the presence of toxins and pathogens. Investigating Lake Iluka is based on data from a large coastal lake in the Illawarra area, Lake Illawarra. Various reports presented on this lake, such as the Lake Illawarra Management Committee (1986), in particular Nr.3, Feb., and those presented by Wollongong City Council (1976), and the State Pollution Control Council, all provide very detailed analysis of the interaction of these factors. It is significant however, that there is general agreement to the notion that, provided the light level and water temperature are within certain accepted tolerances, the most significant controlling factor in the development of eutrophication and hence algal bloom is the availability of nutrients, namely phosphates and nitrates. This simplification can be used to advantage in the development of a 'setmodel' designed to run without interaction.

Such a simplification however would be extremely limiting to the overall educational effectiveness of the package. The important consideration is the complexity of the model upon which the simulation is dependent. Ideally, the simulation will provide a 'better approximation' to the 'real world' if all the interactions between all the limiting factors are taken into consideration. In the earlier stages of research to develop an appropriate 'engine' for this simulation package, AQUASIM developed by Bowker \& Randerson (1989) and a mode developed by Middlebrooks et al. (1975) were considered, however both are considered to be not entirely suited to 
the 'interactive, what if' approach of this package. The use of Stella II ${ }^{\circledR}$ for model development (Figure 2) provides the opportunity to involve all salient factors, however such complexity may prove to be counter productive in terms of the processing load placed on the user by an overly complex interface. The dilemma between over-simplification and excessive complexity will only be fully resolved when the extension to the package is fully field tested. The essential relationships are shown in Figure 1.

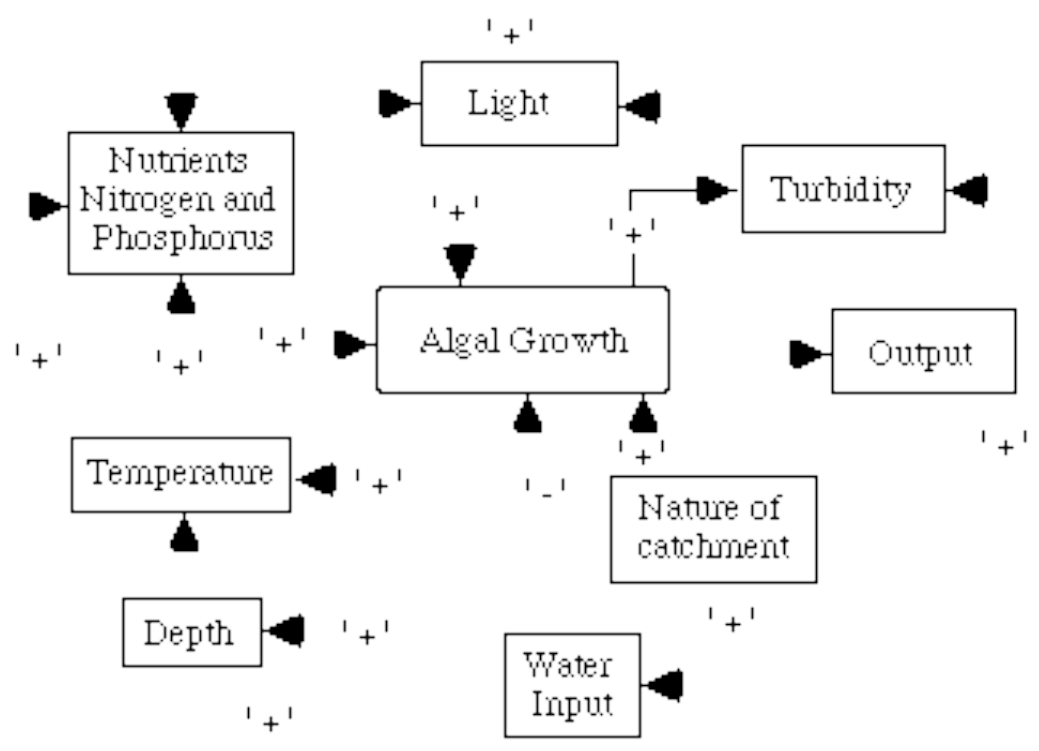

Figure 1: General Relationships

A number of assumptions have been made on which the preliminary design of this simulation model has been based. They have been made after consideration of the research literature on modelling eutrophic processes. Much of this work makes a number of 'standard' assumptions including:

- To reduce the complex interactions so as to maximise the development of understanding, precision will be foregone for realism and generality.

- The body of water which the model depicts is a relatively shallow one.

- The body of water is well mixed. Open shallow lakes have generally evenly distributed nutrient loads. 
- Since algal blooms are limited to the 'eutrophic depth', it may produce an unnecessary complexity to include any direct manipulation of depth.

- Changes in surface algal biomass will be depicted by Chlorophyll(a) mass.

- The variables, $\mathrm{pH}$, bacterium levels, depletion of algae by grazing, and the algal loss due to sinking have negligible effect on total algal biomass.

- Nutrient loads enter and leave the body of water almost exclusively by via water transport.

The basic design considerations included:

- The package is HyperCard driven with QuickTime support.

- This simulation is a 'stand alone', 'plug-in' module to the basic Investigating Lake Iluka package.

- There is a 'set-model' which will provide the user with an indication of the operation of the package. This set-model has a number of starting options based on the nature of the catchment area.

- The interactive simulation has been designed using the modelling software Stella II. It operates on the basis of 'measured input values' embedded in the 'simulated lake' using a 'tool box' containing appropriate instruments or, on the basis of 'what if' values supplied by the user (Figure 2).

- Data input methods depend on the complexity of the model in its final form. The initial parameters over which the user has control (via a tool box) include, temperature, light levels, nutrient input (linked to changes which may be made to the development around the lake including changes in drainage patterns etc., brought about by changes in industrial and urban development). 


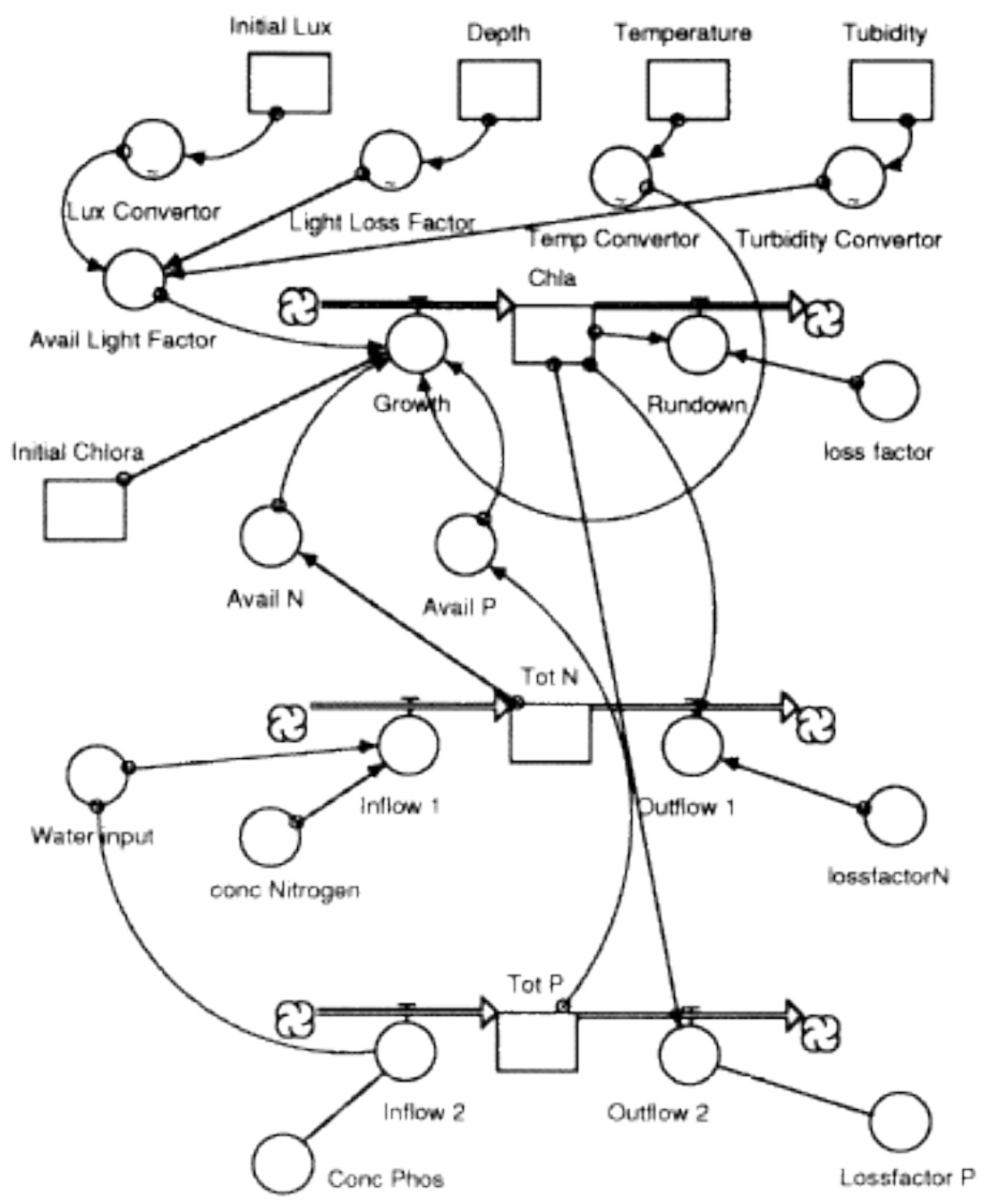

Figure 2: Set-Model

- The simulation has three options (Figure 3); a 'pre-set' option which will operate as a demonstration module, a 'what if' option which will allow the user to collect and use embedded data in the model lake or input independent values for various parameters, and a 'build your own' model, perhaps based on an in-built simplified version of the Stella software. 


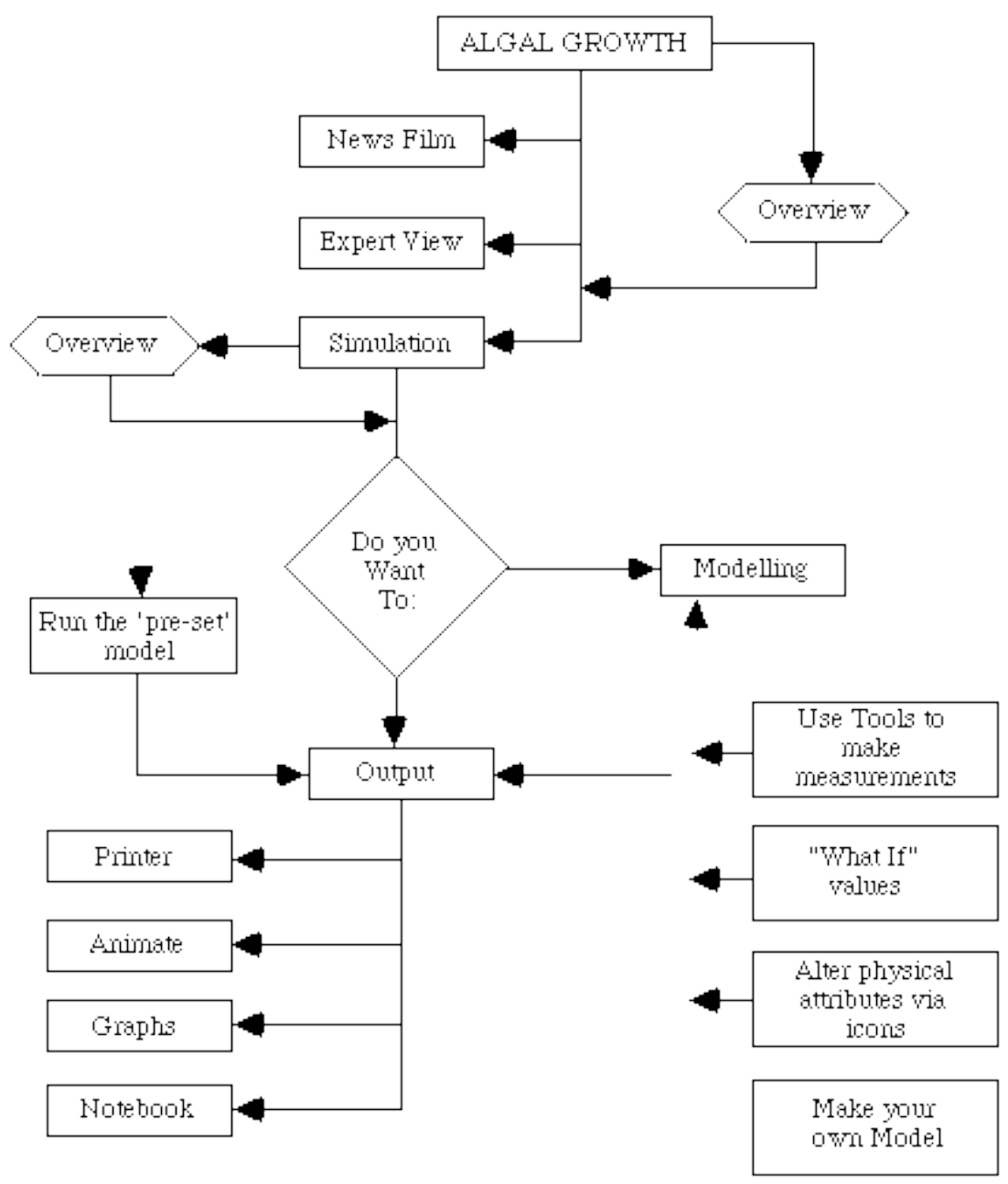

Figure 3: Simulation Flow

The 'what if' and 'build your own' options facilitate more detailed exploration and learning by:

1) allowing the user to take readings at a site and study the changes as the simulation runs,

2) allowing the monitoring of all parameters while the simulation is running, with the aim of exploring the relationships between them. 
Output is via:

1) an animated lake showing the spread/decline of algal mats,

2) graphical presentation of data produced as the simulation runs,

3) the 'tool box' may provide read out simultaneously with displays 1 and 2 ,

4) printer and,

5) cut and paste facilities which allow the storage of data in the student's note book (a feature of the earlier package design) for later manipulation.

\section{Navigation aids}

The educational effectiveness of the package is enhanced by providing the users with 'easy passage', and some indication of where they have been and the choices and decisions they have made. Such information is invaluable to the student's development of higher order skills. The 'feedback on actions' in problem solving situations will not only develop cognitive skills but will also provide a mechanism for the investigation of the cognitive skills involved. Such information may be made available via the student note book.

\section{Learning evaluation}

It is desirable that at any stage of the simulation, the student should be able to determine the effects of decisions made so far on the outcome of the model. To this end, the following information has been provided for optional display.

1) data input so far,

2) the original settings for the area under study,

2) the change in nutrient levels effected as each of the input parameters are altered,

4) the consequent changes in algae density, and

5) a comparison of the current values with some other area in the simulated lake.

\section{Conclusion}

The development of algal blooms in lakes is a process which presents teachers with two major difficulties. The time factor and the collection and monitoring of sufficient data to enable students to analyse the cause and effect relationships would prohibit most teachers presenting such phenomenon in any way other than a superficial and theoretical way. The simulation outlined in this paper seeks to redress this. This simulation takes full advantage of the currently available hardware, and the implications of current research in the field of Cognitive Science. 
The value of simulation packages has been recognised by many researchers. Lebowtz (1986) refers to the instruction method as "explanation-based learning". Nickerson (1988) and Silver (1987) indicate its value as "relating everyday learning to 'real world' based problems, a notion also supported by Pea (1987). Kozma (1991) suggests that the computer can have the capacity to create "dynamic symbolic representations of non-concrete, formal constructs... (and is able to)... proceduralize the relationships between these objects... and... manipulate these representations..." p 199.

Teachers, researchers and indeed the learners themselves (from discussions between the authors and a variety of high school students), have confirmed the notion held by many that good simulations have their relevancy in the provision of experiences that would otherwise not be easily accessible and, are at least as good as 'hands on' experiences. This simulation provides the user with a tool to explore the complexities of ecosystems and the interactions that occur within them. The quality of the experience should match the 'real world' system on which it is based. Although the 'traditional' measure of success may be the acquisition of a knowledge base, it is the ultimate aim of this simulation to achieve a second learning outcome, namely the development of the higher order skills which come with experience in manipulation of the model itself and the observation and analysis of the consequences.

\section{References}

Bell, S. \& Scott, I. (1985). Simulation: Ideas for Using Computers in the Classroom. London: Nelson.

Bliss, J. \& Ogborn, J. (1989). Tools for exploratory learning. Journal of Computer Assisted Learning, 5, 37-50.

Bowker, D. W. \& Randerson, P. F. (1989). A computer simulation of the trophic dynamics of an aquatic system. Journal of Biological Education, 23(1).

Bredemeier, D. W. \& Greenblat, C. S. (1981). The educational effectiveness of simulation games: A synthesis of findings. Simulation $\mathcal{E}$ Games, 12(3), 307-332. Sage Publications.

Crookall, D., Oxford, R. \& Saunders, D. (1987). Towards a reconceptualization of simulation: From representation to reality. Simulation/Games For Learning, 17(4).

Decorte, E. (1990). Learning with new information technologies in schools: Perspectives from the psychology of learning and instruction. Journal of Computer Assisted Learning, 6, 69-87.

Gredler, M. B. (1990). Analysing deep structure in games and simulations. Simulation/Games For Learning, 20(3).

Hooper, K. (1988). Interactive Multimedia Design. Technical Report \#13. Nov., Apple Computer Inc.

Kozma, R. B. (1991). Learning with media. Review of Educational Research, 61(2), 179211.

Lake Illawarra Management Committee (1986). The Nutrient Problem, Nr. 3, Feb.

Laveault, D. \& Corbeil, P. (1990). Assessing the Impact of Simulation Games on Learning: A Step by Step Approach. Simulation/Games For Learning, 20(1). 
Loveluck, C. (1989). Some conceptual changes in micro-computer based games. Simulation/Games For Learning, 19(4).

Martin, A. (1988). Out of the screen: Computers and simulation. Simulation/Games For Learning, 8(1), March.

Middlebrooks, E. J., Fakenburg, D. H. \& Maloney, T. E. (1975). Modelling The Eutrophication Process. New York: Ann Arbor Science Publications.

Nickerson, R. S. (1988). Technology in education in 2020: Thinking about the notdistant future. In R. S. Nickerson \& P. P. Zodhiates (Eds.), Technology In Education: Looking Toward 2020. Hillsdale, NJ: Lawrence Erlbaum Associates.

Ohlsson, S. (1987). Some principles of intelligent tutoring. In R. W. Lawler, \& M. Yazdani (Eds), Artificial Intelligence And Education. Vol. 1. Learning Environments And Tutoring Systems. Norwood, NJ: Ablex Publishing.

Pea, R. (1987). Cognitive technologies for mathematics education. In A. H. Schoenfeld (Ed.), Cognitive Science and Mathematics Education. Hillsdale, NJ: Lawrence Erlbaum Associates.

Reif, F. (1987). Instructional design, cognition and technology: Applications to the teaching of scientific concepts. Journal of Research in Science Teaching, 24(4), 309324.

Reigeluth, C. M. \& Curtis, R. V. (1987). Learning situations and instructional models. In R. M. Gagne (Ed.), Instructional Technology: Foundations. Hillsdale, NJ: Lawrence Erlbaum Associates.

Salomon, G. (1981). Communication and Education: Social and Psychological Interactions. Beverly Hills, CA: Sage Publications.

Schoenfeld, A. H. (1987). What's all the fuss about metacognition? In A. H. Schoenfeld (Ed.), Cognitive Science and Mathematics Education. Hillsdale, NJ: Lawrence Erlbaum Associates.

Silver, E. A. \& Marshall, S. P. (1990). Mathematical and scientific problem solving: Findings, issues and instructional implications. In B. F. Jones, \& L. Idol (Eds.), Dimensions of Thinking and Cognitive Instruction. Hillsdale, NJ: Lawrence Erlbaum Associates.

Silver, E. A. (1987). Foundations of cognitive theory and research for mathematics problem solving instruction. In A. H. Schoenfeld (Ed.), Cognitive Science and Mathematics Education. Hillsdale, NJ: Lawrence Erlbaum Associates.

State Pollution Control Council. (Undated). Lake Illawarra Environmental Audit.

Wollongong City Council and University of Wollongong. (1976). An Environmental Assessment: Lake Illawarra. September. Kiama, NSW: University of Wollongong \& Independent Press.

Authors: Robert Corderoy is a postgraduate research student in the Interactive Multimedia Program at the University of Wollongong. He also coordinates science at a local high School. Drs Barry Harper and John Hedberg teach in the Postgraduate Information Technology Program at University of Wollongong.

Please cite as: Corderoy, R. M., Harper, B. M. and Hedberg, J. G. (1993). Simulating algal bloom in a lake: An interactive multimedia implementation. Australian Journal of Educational Technology, 9(2), 115-129. http: / / www.ascilite.org.au/ajet/ajet9/ corderoy.html 